EESTI NSV TEADUSTE AKADEEMIA TOIMETISED. 29. KOIDE FOUSIKA * MATEMAATIKA. 1980, NR. 2

ИЗВЕСТИЯ АКАДЕМИИ НАУК ЭСТОНСКОП ССР. ТОМ 29

ФИЗИКА * МАТЕМАТИКА, 1980, № 2

Инна РЕБАНЕ, В. ХНЖНЯКОВ

\title{
ИНТЕРФЕРЕНЦИЯ РЕЗОНАНСНОГО И НЕРЕЗОНАНСНОГО РАССЕЯНИЯ СВЕТА В ПРИМЕСНЫХ КРИСТАЛЛАХ
}

\author{
(Представил К. К. Ребане)
}

\section{1. Введение}

Развитая в работах $\left[{ }^{1}\right]$ (см. также обзоры $\left[{ }^{2}\right]$ ) теория резонансного вторичного свечения (РВС) примесных центров кристаллов учитывала вклад в оператор поляризуемости только резонансного электронного уровня (одного или нескольких). Цель настоящей работы - рассмотреть картину вторичного свечения (BC), учитывая наряду с резонансным электронным уровнем и все остальные (нерезонансные). Отметим, что в ряде случаев учет последних весьма существен, поскольку возникающая интерференция вкладов от резонансного и нерезонансных уровней в сечении рассеяния влияет на форму возбуждающих профилей рэлеевской и комбинационных линий.

\section{2. Учет резонансной и нерезонансной поляризуемостей}

Рассмотрим прежде всего интерференцию резонансного и нерезонансного рассеяния света в общем виде. Как известно, сечение рассеяния света в телесный угол $d \Theta$ в спектральный интервал $(\Omega, \Omega+d \Omega)$ определяется формулой

$$
I\left(\omega_{0}, \Omega\right) d \Omega d \Theta=\sum_{\substack{\alpha \alpha^{\prime} \\ \beta \beta^{\prime}}} i_{\alpha} i_{\alpha^{\prime}} n_{\beta} n_{\beta}, W_{\alpha \beta \beta^{\prime} \alpha^{\prime}}\left(\omega_{0}, \Omega\right) d \Theta d \Omega,
$$

где $\omega_{0}$ - частота первичного (возбуждающего) фотона, $i_{\alpha}$ и $n_{\beta}$ - декартовы компоненты единичных векторов поляризации первичного и вторичного фотонов. Тензор четвертого ранга

$$
W_{\alpha \beta \beta^{\prime} \alpha^{\prime}}\left(\omega_{0}, \Omega\right)=B \sum_{i} w_{i} \sum_{f}\left(P_{\alpha \beta}\right)_{i f}\left(P_{\beta^{\prime} \alpha^{\prime}}^{+}\right)_{f i} \delta\left(\omega_{0}-\Omega+E_{i}-E_{f}\right)
$$

есть тензор ВС. Здесь $B=4 \pi^{2} \Omega^{3} \omega_{0} / c^{4}$ и $i, f-$ номера начального $(|i\rangle)$ и конечного $(|f\rangle)$ состояний вещества, $E_{i}\left(E_{f}\right)-$ их энергии, $w_{i}$ - вероятность нахождения вещества в начальном состоянии, тензор

$$
\left(P_{\alpha \beta}\right)_{i f}=\sum_{m}\left(\frac{\left\langle i\left|d_{\alpha}\right| m\right\rangle\left\langle m\left|d_{\beta}\right| f\right\rangle}{E_{m}-E_{i}-\omega_{0}+i \gamma_{m}}+\frac{\left\langle i\left|d_{\beta}\right| m\right\rangle\left\langle m\left|d_{\alpha}\right| f\right\rangle}{E_{m}-E_{f}+\omega_{0}}\right)
$$

называется тензором рассеяния или поляризуемостью перехода $i \rightarrow f$, 
$\gamma_{m}$ - константа радиационного затухания промежуточного состояния $|m\rangle, \vec{d}$ - оператор дипольного момента оптических электронов.

Электронно-колебательные состояния вещества будем описывать в адиабатическом приближенин. В качестве исходных и конечных состояний в (3) выберем колебательные состояния $\left|v_{0}\right\rangle$ и $\left|v_{0}^{\prime}\right\rangle$ основного электронного состояния $|0\rangle:|i\rangle=\left|v_{0}\right\rangle|0\rangle, \quad|f\rangle=\left|v_{0}^{\prime}\right\rangle|0\rangle$; а промежуточные состояния запишем в виде $|m\rangle=\left|v_{e}\right\rangle|e\rangle$. Тогда

$$
\left(P_{\alpha \beta}\right)_{i f}=\left(P_{\alpha \beta, n}\right)_{i f}+\left(P_{\alpha \beta, r}\right)_{i f},
$$

где

$$
\left(P_{\alpha \beta, n}\right)_{i f}=\sum_{e}^{\prime} \sum_{v_{e}}\left(\frac{\left\langle v_{0}\left|M_{\alpha}^{e+}\right| v_{e}\right\rangle\left\langle v_{e}\left|M_{\beta}^{e}\right| v_{0}^{\prime}\right\rangle}{E_{m}-E_{i}-\omega_{0}}+\frac{\left\langle v_{0}\left|M_{\beta}^{e+}\right| v_{e}\right\rangle\left\langle v_{e}\left|M_{\dot{\alpha}}^{e}\right| v_{0}^{\prime}\right\rangle}{E_{m}-E_{f}+\omega_{0}}\right)
$$

и

$$
\left(P_{\alpha \beta, r}\right)_{i f}=\sum_{v_{r}} \frac{\left\langle v_{0}\left|M_{\alpha}^{r+}\right| v_{r}\right\rangle\left\langle v_{r}\left|M_{\beta}^{r}\right| v_{0}^{\prime}\right\rangle}{E_{r}-E_{i}-\omega_{0}+i \gamma_{r}}
$$

- нерезонансный и резонансный тензоры поляризуемости соответственно. Здесь $M_{\alpha} e^{e}=\left\langle e\left|d_{\alpha}\right| 0\right\rangle$ - электронный матричный элемент перехода $|0\rangle \rightarrow|e\rangle$. В (5) штрих у суммы по $e$ означает, что не учтен уровень $|e\rangle=|r\rangle$; в (6) сохранен только первый член из правой части формулы (3), а второй член, описывающий процессы с двумя фотонами в промежуточном состоянии поля, опущен ввиду его малости в резонансном случае по сравнению с первым.

Используя интегральное представление $\delta$-функцин, формулу (2) для тензора ВС можно записать в виде

$$
\left.W_{\alpha \beta \beta^{\prime} \alpha^{\prime}}\left(\omega_{0}, \Omega\right)=B /(2 \pi) \int_{-\infty}^{\infty} d \mu \mathrm{e}^{-i\left(\omega_{0}-\Omega \pi \mu^{\prime} \gamma\right.} P_{\alpha \beta^{\prime}}(\theta) P_{\beta^{\prime} \alpha^{\prime}}^{+}(\mu)\right\rangle_{0}, \varepsilon
$$

где $\langle\ldots\rangle=\sum_{v_{0}} w_{v_{0}}\left\langle v_{0}|\ldots| v_{0}\right\rangle=\mathrm{Sp}\left(\mathrm{e}^{-H_{0} / k T} \ldots\right) / \mathrm{Sp}\left(\mathrm{e}^{-H^{2} / k T}\right)-$ знак статистического усреднения по исходным колебательным состояниям; $P_{\alpha \beta}(\mu)=$ $=\exp \left(i \mu H_{0}\right) P_{\alpha \beta} \exp \left(-i \mu H_{0}\right) ; H_{0}-$ колебательный гамильтониан исходного электронного состояния: $\left.\left.H_{0}\right\rfloor v_{0}\right\rangle=E_{v_{0}}\left|v_{0}\right\rangle ; P_{\alpha \beta}$ - оператор поляризуемости с матричными элементами $\left\langle v_{0}\left|P_{\alpha \beta}\right| v_{0}^{\prime}\right\rangle=\left(P_{\alpha \beta}\right)_{v_{0} v_{0}^{\prime}}=\left(P_{\alpha \beta}\right)_{i f}$.

Ниже для резонансного оператора поляризуемости основное $|0\rangle$ и возбужденное $|r\rangle$ электронные состояния принимаются невырожденными, используется приближение Кондона: $M_{\alpha}^{r}, M_{\beta}^{r}=$ const, $\gamma_{r}=$ $=\gamma=$ const и предполагается, что колебательные гамильтонианы $H_{0}$ и $H_{r}$ отличаются лишь положением равновесия ядер. В этом случае резонансный оператор поляризуемости имеет следующий вид:

$$
P_{\alpha \beta, r}=-i M_{\alpha}^{r+} M_{\beta}^{r} \int_{0}^{\infty} d \tau \mathrm{e}^{i\left(\omega_{1}-\omega_{0}\right) \tau-\gamma \tau} T \mathrm{e}^{\nabla(-\tau)-\nabla} .
$$

Здесь

$$
\nabla(x)=\sum_{j} \xi_{0 j}\left(a_{j} \mathrm{e}^{-i \omega_{1} x}-a_{j}^{+} \mathrm{e}^{i \omega_{j} x}\right), \quad, \quad,
$$

$\xi_{0 j}{ }^{2}$ - безразмерные стоксовы потери, приходящиеся на осциллятор $j$; 
$a_{j}^{+}$и $a_{j}$ - операторы рождения и уничтожения фонона частоты $\omega_{j}$; $\omega_{1}$ - частота чисто электронного перехода; $T$ - символ упорядочения операторов в момент времени - $\tau$ слева от операторов в момент времени ноль. Как видно, в случае невырожденных основного и возбужденного (резонансного) электронных состояний оператор поляризуемости четный: $P_{\alpha \beta, r}=P_{\beta \alpha, r}$.

Для нерезонансного оператора поляризуемости предположим, как обычно, что разности энергий электронных переходов и $\omega_{0}$ много больше колебательной энергии:

$$
\begin{aligned}
& E_{m}-E_{i}-\omega_{0}=\left(E_{e}+E_{v_{e}}\right)-\left(E_{0}+E_{v_{0}}\right)-\omega_{0} \simeq-\omega_{0 e}-\omega_{0}, \\
& E_{m}-E_{f}+\omega_{0}=\left(E_{e}+E_{v_{e}}\right)-\left(E_{0}+E_{v_{0}^{\prime}}\right)+\omega_{0} \simeq-\omega_{0 e}+\omega_{0} \\
& \left(\omega_{0 e} \equiv E_{0}-E_{e}\right) .
\end{aligned}
$$

Тогда

$$
P_{\alpha \beta, n} \simeq-\sum_{e}^{\prime}\left(\frac{\sum_{v_{e}} M_{\alpha}^{e+}\left|v_{e}\right\rangle\left\langle v_{e}\right| M_{\beta}^{e}}{\omega_{0 e}+\omega_{0}}+\frac{\sum_{v_{e}} M_{\beta}^{e+}\left|v_{e}\right\rangle\left\langle v_{e}\right| M_{\alpha}^{e}}{\omega_{0 e}-\omega_{0}}\right)
$$

Предполагая, далее, что электронные матричные элементы $M_{\alpha}{ }^{e}, M_{\beta}{ }^{e}$ зависят только от колебательных координат основного электронного состояния (т. е. не зависят от координат промежуточных состояний), запишем нерезонансный оператор поляризуемости в виде

$$
P_{\alpha \beta, n}=-\Sigma_{e}^{\prime} \frac{2 \omega_{0 e} M_{\alpha}^{e+} M_{\beta}^{e}}{\omega_{0 e}^{2}-\omega_{0}^{2}} .
$$

Хотя, вообще говоря, тензор $P_{\alpha \beta, n}$ несимметричен $\left[{ }^{3}\right]$, однако в приближении (11) он, как и тензор $P_{\alpha \beta, r}$, симметричен.

Разложим $P_{\alpha \beta, n}$ в ряд по безразмерным нормальыы координатам $\xi_{j}(x)=a_{j} \mathrm{e}^{-i \omega_{l} x}+a_{j}^{+} \mathrm{e}^{i \omega_{j} x}$ :

$$
P_{\alpha \beta, n}=P_{\alpha \beta, n, 0}+\sum_{k=1}^{\infty}(1 / k !) P_{\alpha \beta, n, i_{1} \ldots i_{k}}\left(\prod_{r=1}^{k} \xi_{i_{r}}\right) .
$$

В разложении (12) члены $k$-го порядка по $\xi$ соответствуют комбинационному рассеянию $k$-го порядка. Ниже мы ограничимся рассмотрением рэлеевского и комбинационного, рассеяния первого порядка. В таком случае

$$
P_{\alpha \beta, n} \simeq P_{\alpha \beta, n, 0}+\sum_{i} P_{\alpha \beta, n, i} \xi_{i}
$$

где

$$
\begin{gathered}
P_{\alpha \beta, n, 0}=-\sum_{e}^{\prime} \frac{2 \omega_{0 e}}{\omega_{0 e}^{2}-\omega_{0}^{2}}\left(M_{\alpha}^{e+} M_{\beta}^{e}\right)_{0}, \\
P_{\alpha \beta, n, i}=-\sum_{e}^{\prime} \frac{2 \omega_{0 e}}{\omega_{0 e}^{2}-\omega_{0}^{2}}\left(\frac{\partial\left(M_{\alpha}^{e+} M_{\beta}^{e}\right)}{\partial \xi_{i}}\right)_{\left\{\xi_{l}\right\}=0} .
\end{gathered}
$$

Следует подчеркнуть, что последовательная теория рэлеевского и ком- 
бинационного рассеяния должна учитывать не только поляризуемость примесного центра, но и поляризуемость кристалла-основания. Поэтому в формуле (12) под $P_{\alpha \beta, n}$ следует понимать нерезонансную часть поляризуемости всего кристалла, включая центр.

\section{3. Сечение рассеяния. Возбуждающие профили}

Для получения тензора ВС (7) необходимо вычислить коррелятор

$$
\begin{gathered}
\left\langle P_{\alpha \beta} P_{\beta^{\prime} \alpha^{\prime}}^{+}(\mu)\right\rangle_{0}=\left\langle P_{\alpha \beta, r} P_{\beta^{\prime} \alpha^{\prime}, r}^{+}(\mu)\right\rangle_{0}+\left\langle P_{\alpha \beta, r} P_{\beta^{\prime} \alpha^{\prime}, n}^{+}(\mu)\right\rangle_{0}+ \\
+\left\langle P_{\alpha \beta, n} P_{\beta^{\prime} \alpha^{\prime}, r}^{+}(\mu)\right\rangle_{0}+\left\langle P_{\alpha \beta, n} P_{\beta^{\prime} \alpha^{\prime}, n}^{+}(\mu)\right\rangle_{0} .
\end{gathered}
$$

Подставляя (16) в (7), получаем, что первый член в правой части формулы (16) описывает РВС, последний - нерезонансное ВС (НВC), а два средних учитывают интерференцию РВС и НВС. Используя разложение операторов $P_{\alpha \beta, r}$ и $P_{\alpha \beta, n}$ по $a_{j}$ и $a_{j}{ }^{+}$, можно сечение всего ВС представить в виде разложения по порядку $(s)$ рассеяния

$$
I\left(\omega_{0}, \Omega\right)=\sum_{s} I_{s}\left(\omega_{0}, \Omega\right)=\sum_{s}\left[I_{r, s}\left(\omega_{0}, \Omega\right)+I_{i, s}\left(\omega_{0}, \Omega\right)+I_{n, s}\left(\omega_{0}, \Omega\right)\right] .
$$

Следует отметить, что фигурирующие в (17) парциальные спектры РВС описывают все компоненты ВС: резонансное рэлеевское и резонансное комбинационное рассеяние (РКР), а также горячую и обычную люминесценцию $\left[{ }^{2}\right]$, а парциальные спектры НВС - лишь рэлеевское и нерезонансное комбинационное рассеяние (НКР). Член нулевого порядка в (17), описывающий рэлеевское рассеяние, равен

$$
\begin{gathered}
I_{0}\left(\omega_{0}, \Omega\right)=\left.B|| M^{r}\right|^{2} \Phi\left(\omega_{0}\right)+\left.P_{n, 0}\right|^{2} \delta\left(\omega_{0}-\Omega\right) \\
\left|M^{r}\right|^{2} \equiv \sum_{\alpha, \beta} i_{\alpha} n_{\beta} M_{\alpha}^{r+} M_{\beta}^{r}=\sum_{\alpha^{\prime}, \beta^{\prime}} i_{\alpha^{\prime}} n_{\beta} \cdot M_{\beta^{\prime}}^{r+} M_{\alpha^{\prime}}^{r}, \\
P_{n, 0} \equiv \sum_{\alpha, \beta} i_{\alpha} n_{\beta} P_{\alpha \beta, n, 0}=\sum_{\alpha^{\prime}, \beta^{\prime}} i_{\alpha^{\prime}} n_{\beta^{\prime}} P_{\beta^{\prime} \alpha^{\prime}, n, \boldsymbol{\theta}} \\
\Phi(\omega)=-i \int_{0}^{\infty} d \tau \mathrm{e}^{i\left(\omega_{1}-\omega\right) \tau-\gamma \tau+g(\tau)} .
\end{gathered}
$$

Функция

$$
g(x)=\left\langle\nabla^{2}\right\rangle_{0}-\langle\nabla \nabla(x)\rangle_{0}
$$

определяет логарифм Фурье-образа спектров поглощения и люминесценции $\left[{ }^{4}\right]$. В гармоническом приближении эта функция равна

$$
g(x)=\sum_{j} \xi_{0 j}^{2}\left[\left(\mathrm{e}^{i \omega_{j} x}-1\right)\left(\bar{n}_{j}+1\right)+\left(\mathrm{e}^{-i \omega_{j} x}-1\right) \bar{n}_{j}\right]
$$

где $\bar{n}_{j}=\left[\exp \left(\omega_{j}^{\prime} k T\right)-1\right]^{-1}-$ среднее число фононов частоты $\omega_{j}$ при температуре $T$.

Как видно из формулы (18), в случае парциального спектра нулевого порядка пронсходит сложение амплитуд вероятностей резонансного и нерезонансного рассеяния, что приводит к эффекту интерференции последних. Парцнальный спектр первого порядка 
Возбуждающие профили: нерезонансного рассеяния $(1)$, резонансного рассеяния (2), с учетом интерференции резонансного и нерезонансного рассеяния в случае суммы (3) и разности (4) амплитуд. Параметры: $\sigma=$ $=0,02 \omega_{0 r}, \quad \overrightarrow{\omega_{0 e}}=1,5 \omega_{0 r}, \quad f_{r_{0}}=0,1 \quad$ и $\alpha_{i} / \beta_{i}=0,1$ (3) или $\alpha_{i} / \beta_{i}=-0,1$ (4).

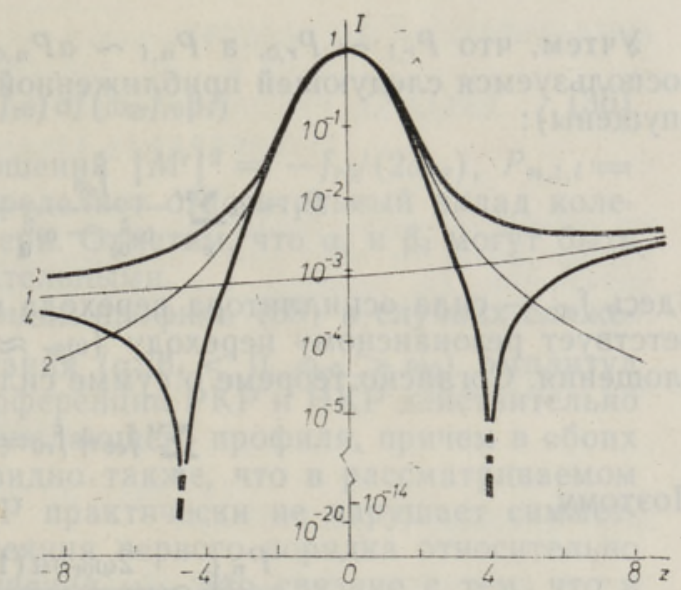

$$
I_{1}\left(\omega_{0}, \Omega\right)=B \sum_{i}\left\{( \overline { n } _ { i } + 1 ) \delta ( \Omega - \omega _ { 0 } + \omega _ { i } ) \left\lfloor| M ^ { r } | ^ { 2 } \xi _ { 0 i } \left[\Phi\left(\omega_{0}\right)-\right.\right.\right.
$$

$\left.-\Phi(\Omega)]-\left.P_{n, i}\right|^{2}+\bar{n}_{i} \delta\left(\Omega-\omega_{0}-\omega_{i}\right)|| M^{r}\left|{ }^{2} \xi_{0 i}\left[\Phi\left(\omega_{0}\right)-\Phi(\Omega)\right]+P_{n, i}\right|^{2}\right\}$, где

$$
P_{n, i} \equiv \sum_{\alpha, \beta} i_{\alpha} n_{\beta} P_{\alpha \beta, n, i}=\sum_{\alpha^{\prime}, \beta^{\prime}} i_{\alpha^{\prime}} n_{\beta^{\prime}} P_{\beta^{\prime} \alpha^{\prime}, n, i}
$$

Для анализа возбуждающего профиля используем разложение Ф $(\Omega) \simeq$ $\simeq \Phi\left(\omega_{0}\right) \mp \omega_{i} \Phi^{\prime}\left(\omega_{0}\right)$, где знак «минус» соответствует стоксовой части спектра, знак «плюс»- антистоксовой. Тогда

$$
\begin{aligned}
& I_{1}\left(\omega_{0}, \Omega\right)=B \sum_{j}\left\{\left[\left(\bar{n}_{i}+1\right) \delta\left(\Omega-\omega_{0}+\omega_{i}\right)+\right.\right. \\
& \left.\left.+\bar{n}_{i} \delta\left(\Omega-\omega_{0}-\omega_{i}\right)\right]\left.|| M^{r}\right|^{2 \xi_{i 0} \omega i} \Phi^{\prime}\left(\omega_{0}\right)-\left.P_{n, i}\right|^{2}\right\}
\end{aligned}
$$

Здесь возможны два случая (см. рисунок) : действительная часть амплитуды резонансного рассеяния и амплитуда нерезонансного рассеяния (которая целиком действительна) имеют либо одинаковые знаки (3), либо разные (4). Из рисунка видно, что в этих случаях возбуждающие профили существенно различаются. Знаки амплитуд зависят от вида функции $Ф(\omega)$, знака сдвига положения равновесия छㅇ, знака производной $\left[\partial\left(M_{\alpha}^{e+} M_{\beta}^{e}\right) / \partial \xi_{i}\right]_{\left\{\xi_{\}}\right\}=0}$ и знака разности $\omega_{0 e}{ }^{2}-\omega_{0}^{2}$. Сложение или вычитание амплитуд происходит для каждого нормального колебания в отдельности. При этом эффект интерференции рассеяний возможен лишь на тех колебаниях, которые дают вклад как в резонансное рассеяние, так и в нерезонансное. В рассматриваемом случае резонансного перехода между невырожденными уровнями вклад в РВС дают только полносимметричные колебания. Поэтому и интерференция имеет место только для рассеяния на полносимметрғчных колебаниях.

\section{4. Оценки. Заключительные замечания}

Сравним вклады резонансного и нерезонансных уровней в сечение рассеяния первого порядка на колебаниях примесного центра. В этом случае вклад в рассеяние будет давать только та часть поляризуемости, которая связана с центрами. 
Учтем, что $P_{r, 1} \sim P_{r, 0}$, а $P_{n, i} \sim \alpha P_{n, 0}$, где обычно $\alpha \sim 0,1-0,01$, и воспользуемся следующей приближенной формулой (тензорные индексы опущены):

$$
P_{1} \sim \alpha \sum_{e}^{\prime} \frac{f_{e 0}}{\omega_{0 e}^{2}-\omega_{0}^{2}}+\frac{f_{r 0}}{2 \omega_{0 r} \sigma} .
$$

Здесь $f_{e 0}-$ сила осциллятора перехода между уровнями 0 и $e, f_{r 0}$ соответствует резонансному переходу $\left(\omega_{0} \approx \omega_{0}\right), \sigma-$ ширина спектра поглощения. Согласно теореме о сумме сил осцилляторов,

Поэтому

$$
\sum_{e}^{\prime} f_{e 0}+f_{r 0}=1
$$

$$
\frac{P_{n, 1}}{P_{r, 1}} \sim \frac{2 \omega_{0 r} \sigma \alpha\left(1-f_{r 0}\right)}{f_{r 0}\left(\bar{\omega}_{0 e}^{2}-\omega_{0 r}^{2}\right)},
$$

где $\bar{\omega}_{0 e}-$ среднее значение частот электронных переходов $\omega_{0 е}$. Например, при $\sigma=0,02 \omega_{0 r}, \omega_{0 e}=1,5 \omega_{0 r}$ и $f_{r 0}=0,1$ величина $P_{n, 1} / P_{r, 1} \sim 0,03$.

Вклад НВС в высшие порядки парциальных спектров обычно очень мал и им можно пренебречь.

Для получения возбуждающего профиля рассеяния первого порядка воспользуемся формулой

$$
\Phi(\omega)=P \int d x x(x) /(\omega-x)-i \pi x(\omega),
$$

где $x\left(\omega_{0}\right)$ - спектр поглощения. Зададим его в виде нормального распределения

$$
x\left(\omega_{0}\right)=\mathrm{e}^{-z^{2}} /(\sqrt{2 \pi} \sigma),
$$

где

$$
z=\left(\omega_{0}-\omega_{0 r}\right) /\left(\sqrt{2}^{r} \sigma\right)
$$

- безразмерная частота возбуждения, $\sigma^{2}$ - второй центральный момент спектра. Это приближение хорошо выполняется для центров с сильным тепловыделением. В этом случае [ $\left.{ }^{1}\right]$

$$
\Phi\left(\omega_{0}\right)=(\sqrt{2} / \sigma)\left[w(z)-i(\sqrt{\pi} / 2) \mathrm{e}^{-z^{2}}\right],
$$

где

$$
w(z)=\mathrm{e}^{-z^{2}} \int_{0}^{z} \mathrm{e}^{x^{2}} d x
$$

- функция Даусона, табулированная в [5].

Подставляя (33) в (26), находим возбуждающий профиль для $i$-го колебания

$$
\begin{gathered}
I_{1, i}\left(\omega_{0}\right)=\left.|| M^{r}\right|^{2 \xi} \xi_{0 i} \omega i \frac{\Phi^{\prime}(z)}{\sqrt{2} \sigma}-\left.P_{n, i}\right|^{2} \simeq \\
\simeq\left|w^{\prime}(z)+i \sqrt{\pi} z \mathrm{e}^{-z^{2}}+C \frac{\omega_{0 r}^{2}}{\overline{\omega_{0 e}^{2}}-\left(\sqrt{2} \sigma z+\omega_{0 r}\right)^{2}}\right|^{2},
\end{gathered}
$$


где

$$
C=2 \alpha_{i}\left(1-f_{r 0}\right) \sigma /\left(\omega_{0 r} f_{r 0} \beta_{i}\right)
$$

при учете формулы (28) и соотношений $\left|M^{r}\right|^{2}=-f_{r 0} /\left(2 \omega_{0 r}\right), P_{n, 1, i}=$ $=\alpha_{i} P_{n, 0}$ и $\xi_{0 i}=\beta_{i} \sigma / \omega_{i}$, где $\beta_{i}{ }^{2}$ определяет относительный вклад колебания $i$ в суммарные стоксовы потери. Отметим, что $\alpha_{i}$ и $\beta_{i}$ могут быть как положительными, так и отрицательными.

На рисунке показан возбуждающий профиль (35) в случаях сложения $\left(\alpha_{i} / \beta_{i}>0, \vec{\omega}_{e 0}>\omega_{0}\right)$ и вычитания $\left(\alpha_{i} / \beta_{i}<0, \bar{\omega}_{e 0}>\omega_{0}\right)$ амплитуд рассеяния. Видно, что эффект интерференции РКР и НКР действительно существенно влияет на форму возбуждающего профиля, причем в обоих случаях по-разному. Из рисунка видно также, что в рассматриваемом случае интерференция РКР и НКР практически не нарушает симметрию возбуждающего контура рассеяния первого порядка относительно частоты максимума полосы поглощения $\omega_{0}$. Это связано с тем, что в случае НКР амплитуда рассеяния целиком действительна. Поэтому интерферирует только действительная часть амплитуды РКР, которая является четной функцией $z$. Отметим, что указанная симметрия нарушается, если имеются два близко расположенных электронных уровня, полосы поглощения которых перекрываются. В этом случае интерферировать будут как действительные, так и мнимые части амплитуд рассеяння. Последние же в случае рассеяния первого порядка являются нечетными функциями частоты $z$.

Отметим в заключение, что асимметрия возбуждающего профиля может быть вызвана также отклонением от приближения Кондона для резонансного перехода [ $\left.{ }^{6}\right]$.

\title{
Л ИТ Р А Т РА
}

1. Hizhnyakov, V., T ehver, I., Phys. Status Solidi, 21, № 2, 755-768 (1967); Hizhnyakov, V. V., Rebane, K. K., T ehver, I. J., In: Light Scattering Spectra of Solids, ed. G. F. Wright, Springer, New York, 1969, p. 513-519; Hizhnyakov, V., Tehver, I., In: Physics of Impurity Centres in Crystals, Tallinn, 1972, p. 607-626.

2. Reba ne, K., Hizhnyakov, V., Tehver, I., ENSV TA Toim., Füüs. Matem., 16, № 2, 207-232 (1967); Р е б ан е К. К., Х и жня я ов В. В., В кн.: Дж. Би рм ан. Пространственная симметрия и оптические свойства твердых тел, т. 2, М., «Мнр», 1978 , с. $327-350$.

3. О в а н де е Л. Н., Оптика и спектроскопия, 9, вып. 5, 571-575 (1960).

4. L a x, M., J. Chem. Phys., 20, 1752 (1952); (русский перевод см. в кн.: Проблемы физики полупроводников, М., Изд-во иностр. лит., 1957 , с. $407-423)$.

5. Карпов К. А., Таблицы функции $w(z)=\mathrm{e}^{-z^{2}} \int_{0}^{z} \mathrm{e}^{x^{2}} d x$ в комплексной области, М., Изд-во АН СССР, 1954.

6. Техв ер И., Изв. АН ЭССР, Физ. Матем., 17, № 2, 235-238 (1968).

\author{
Институт физики \\ Академии наук Эстонской ССР
}

Поступила в редакцию 24/X 1979 
INNA REBANE, V. HIZNJAKOV

\section{VALGUSE RESONANTSE JA MITTERESONANTSE HAJUMISE INTERFERENTS LISANDIKRISTALLIDES}

On esitatud resonantse kombinatsioonhajumise teooria, mis arvestab resonantse elektronseisundi kõrval ka kõigi teiste (mitteresonantsete) seisundite panust polariseeritavuse operaatorisse. On vaadeldud nimetatud seisundite panuste interferentsi mõju hajumise ristlōikes Rayleigh' ja kombinatsioonhajumise joonte ergastusprofiilide kujule.

INNA REBANE, V. HIZHNYAKOV

\section{THE INTERFERENCE OF RESONANT AND NON-RESONANT SCATTERING IN IMPURITY CENTRES}

An excitational profile of resonant Raman scattering of an impurity centre is invesligated with regard to the contributions of the resonant as well as non-resonant electronic transitions into the polarizability operator. The vibronic states of the centre are described in an adiabatic approximation. Lattice vibrations are considered to be harmonic. For the resonant polarizability operator the Condon approximation is used, the ground and excited electronic states are taken as non-degenerate, and the shift of the nuclear equilibrium positions at an electronic transition are taken into account.

In the present case the probability amplitude of the Raman scattering consists of two terms, one of which takes account of the resonant scattering and the other, of the non-resonant one. The dependence of these two terms on the exciting frequency differs essentially, which results in an appearance of specific interference peculiarities in the excitational profiles of the Raman scattering.

In the case of the first-order partial spectrum there are two interference possibilities for each normal mode: the probability amplitudes to be summed can be of the same or of opposite signs. The «relative contributions» of the resonant and non-resonant parts of the polarizability into the first-order Raman scattering are estimated and the sharps of the excitational profiles for these two possible cases are calculated. 